\title{
Disruptions of preparatory attention contribute to failures of prospective memory
}

\author{
ROBERT WEST, JASON KROMPINGER, and RITVIJ BOWRY \\ University of Notre Dame, Notre Dame, Indiana
}

\begin{abstract}
In two experiments, we examine the efficacy of preparatory attentional processes and intention superiority as explanations of the finding that response time (RT) for prospective memory (PM) cue misses is often faster than that for ongoing activity trials or PM cue hits. RT was faster for prospective misses than for ongoing activity trials, demonstrating an intention superiority effect. RT was also faster for ongoing activity trials preceding prospective misses than for those preceding prospective hits. We interpret this finding as reflecting variation in the efficiency of preparatory attentional processes during task performance, leading to failures of PM when preparatory attentional processes are suboptimal.
\end{abstract}

In the course of an average day, we often find that the realization of many of our goals or intentions must be postponed until such time as an appropriate cue is encountered. The realization of delayed intentions or prospective memory (PM; Ellis, 1996), can be distinguished from working memory (WM), which involves the active maintenance of a limited amount of information for a brief period of time (Baddeley, 1986) and recognition memory, which involves the retrieval of previously experienced episodes (Tulving, 1983). In the typical event-based PM task, individuals are engaged in an ongoing activity that is relatively continuous (e.g., a semantic judgment or lexical-decision task) and are required to make a prospective response (e.g., pressing a designated key on the keyboard) when a prospective cue is encountered during the ongoing activity.

In a recent study examining the processes underlying PM, Marsh, Hicks, and Watson (2002) had individuals make ongoing activity responses that required lexical decisions for words and nonwords on all trials and an additional prospective response on prospective cue trials. Across a series of experiments, response time (RT) was faster for prospective misses on trials in which a prospective cue failed to elicit a prospective response (where only a lexical decision was made) than for ongoing activity trials (where a lexical decision was made) or for prospective hits (where the lexical decision was followed by a prospective response). The faster RTs for prospective misses than for ongoing activity trials was attributed to the expression of the intention superiority effect, which refers to the tendency of RT for recognition judgments and lexical decisions to be faster for words that are related to active intentions than for a variety of other stimuli. The effect is thought to result from the persistence of above-baseline activation of information related to intentions (Goschke \& Kuhl, 1993; Marsh, Hicks, \&

Correspondence should be addressed to R. West, 118 Haggar Hall, University of Notre Dame, Notre Dame, IN 46556 (e-mail: west.19@nd.edu).
Bink, 1998). The present study was designed to extend the findings of Marsh et al. (2002) to a novel paradigm and to examine the ability of two constructs (i.e., preparatory attentional processes and intention superiority) to account for patterns of RT differentiating realized intentions from failures of PM.

Within the preparatory attentional and memory (PAM) processes theory of PM, Smith and Bayen (2004) propose that preparatory attentional processes serve to maintain an attentive state that facilitates the processing of prospective cues when they are encountered in the environment. One piece of evidence offered as support for the existence of preparatory attentional processes is that RT slows down for ongoing activity trials when a PM load is introduced into the task relative to when no PM load is present (Marsh, Hicks, Cook, Hansen, \& Pallos, 2003; Smith, 2003). This slowing of RT for ongoing activity trials with the addition of a prospective load is negatively correlated with WM capacity (Smith, 2003), which is consistent with evidence indicating that the efficiency of PM decreases when the ongoing activity places high demands on the central executive of WM (Marsh \& Hicks, 1998).

The preparatory attentional processes and intention superiority accounts provide alternative explanations for the empirical finding of differences in RT between prospective misses and ongoing activity trials, which are difficult to distinguish on the basis of available evidence. Within the context of PAM, faster RTs for prospective misses in comparison with ongoing activity trials would result from a disruption of preparatory attentional processes. In contrast, based upon the intention superiority account, this difference would arise from the expression of intention superiority in combination with the disruption of other processes supporting PM (Marsh et al., 2002). One means of distinguishing between the preparatory attentional processes and intention superiority accounts is to consider RTs not only for prospective misses, ongoing activity trials, and prospective hits but also for the trials preceding prospective hits and misses. If the 
difference in RT for prospective misses in comparison with to ongoing activity trials results from a disruption of preparatory attentional processes, one would expect RT for ongoing activity trials preceding misses to be faster than that for ongoing activity trials preceding hits, reflecting the waning of preparatory attentional processes. In contrast, if intention superiority produces this difference, faster RTs should be limited to the processing of prospective cues, since information related to cues is thought to be processed more fluently (Goschke \& Kuhl, 1993).

\section{EXPERIMENT 1}

To examine predictions related to preparatory attentional processes and intention superiority, participants performed an $n$-back WM task that required 1-back or 3back judgments and had an embedded PM component. If disruptions of preparatory attentional processes contribute to RT on prospective misses, we would expect RT for ongoing activity trials preceding prospective misses to be faster than that for trials preceding prospective hits. In contrast, if faster RTs for prospective misses reflect the expression of intention superiority, we would expect RTs for prospective misses to be faster than those for ongoing activity trials that were not in close proximity to prospective misses. If preparatory attentional processes depend on the availability of WM capacity (Smith, 2003), we would expect disruptions of preparatory attention to differ in the 1-back and 3-back conditions.

\section{Method}

Participants. Eighteen individuals (6 female; $18-24$ years of age) participated in the study for course credit or a modest cash stipend. Data for 2 participants with no prospective misses were excluded from the analyses.

Materials and Procedure. The stimuli were 21 consonants from the English alphabet presented in yellow. They were displayed in uppercase letters, $15 \times 10 \mathrm{~mm}$, and were viewed from approximately $50 \mathrm{~cm}$. Each letter was displayed for $500 \mathrm{msec}$, followed by a blank screen for $1,500 \mathrm{msec}$.

The task included 12 blocks of trials ( 2 practice and 10 test) that were equally divided between the 1 - and 3-back conditions and three types of trials (i.e., prospective cues on $10 \%$, targets on $30 \%$, and nontargets on $60 \%$ ). The 1 - and 3-back conditions were counterbalanced across participants. Practice blocks included 20 trials, and test blocks included 100 trials. For all blocks, the prospective cues were the letters $\mathrm{D}$ and $\mathrm{M}$. For the 1-back condition, targets represented the second letter of a repetition in a series of letters (e.g., S P P); for the 3-back condition, targets represented the second letter of a repetition following two intervening letters (e.g., L J Q $L$ ). For the 1- and 3-back conditions, nontargets were those trials that did not represent 1- or 3-back repetitions or prospective cues. For the 1-back condition, the participants were instructed to press the $\mathrm{V}$ key (left index finger) on the keyboard when the current letter matched the letter that was presented one back in the sequence and the $\mathrm{N}$ key (right index finger) when the current letter did not match the previous letter; for the 3-back condition, the participants were instructed to press the V key when the current letter matched the letter that was presented three back in the sequence and the $\mathrm{N}$ key when the current letter did not match the 3-back letter. For half of the participants, the target-nontarget key mapping was reversed. For all blocks, they were instructed to press the $\mathrm{C}$ key (left middle finger) when the letter D was presented and the M key (right middle finger) when the letter $M$ was presented, instead of making a target-nontarget response.

\section{Results}

For all analyses, the significance level was set at $p<$ .05 . The proportion of correct responses was lower in the 3-back than in the 1-back blocks. This effect was significant for targets [1-back, $M=88 \%$; 3-back, $M=75 \%$; $t(15)=4.28$ ] and nontargets [1-back, $M=97 \%$; 3-back, $M=90 \% ; t(15)=3.24]$, but not prospective cues [1back, $M=85 \%$; 3-back, $M=82 \% ; t(15)=1.01]$.

To examine hypotheses related to preparatory attentional processes and intention superiority, we considered mean RTs for prospective cue hits and misses, nontarget correct rejections immediately preceding target hits, ${ }^{1}$ and the three trials preceding prospective cue and $n$-back target hits and misses (Figure 1A). RT was faster for prospective misses than for prospective hits $[F(1,15)=$ $\left.4.58, \eta^{2}=.23\right]$, and this effect did not interact with $n$ back load $(F<1)$. RT was also faster for prospective misses $(M=616 \mathrm{msec})$ than for correct nontarget trials preceding target hits $(M=677 \mathrm{msec})$, revealing an intention superiority effect. This difference was not significant in the raw RT data $\left[F(1,15)=1.30, \eta^{2}=.08\right]$ but was significant when the data were log-transformed, reducing the influence of extreme values $[F(1,15)=$ $\left.4.45, \eta^{2}=.23\right]$. RT was faster for the three ongoing activity trials preceding prospective misses than for those preceding prospective hits $\left[F(1,15)=27.94, \eta^{2}=.65\right]$, which is consistent with the prediction that disruptions of preparatory attentional processes contribute to RT for prospective misses. In this analysis, the accuracy $\times$ trial interaction was not significant $(F<1)$, indicating that disruptions of preparatory attentional processes were present at least $6 \mathrm{sec}$ before the presentation of the missed cues.

The RT profiles preceding target hits and misses were also considered, in order to examine similarities in and differences between the processes contributing to PM and WM errors. In this analysis, the accuracy $\times$ load $\times$ trial interaction was significant $\left[F(2,30)=5.23, \eta^{2}=\right.$ .26 ; see Figure 1B]. This interaction reflected nonsignificant differences in RT for the third trial before a target hit or miss [1-back, $t(15)=-.28 ; 3$-back, $t(15)=1.62$ ] and faster RTs for the two trials before misses than for those before hits in the 1-back condition $[t(15)=-3.99$, and $t(15)=-3.91]$ and slower RTs for the two trials preceding misses than for the two trials preceding hits in the 3 -back condition $[t(15)=3.04$, and $t(15)=2.42]$. Differences in the pattern of RT preceding hits and misses for prospective cues and $n$-back targets indicates that different processes contributed to failures of PM and WM.

\section{EXPERIMENT 2}

The results of Experiment 1 indicate that intention superiority and disruptions of preparatory attentional processes contribute to RT for prospective misses. Given the 
(A) Prospective Cues

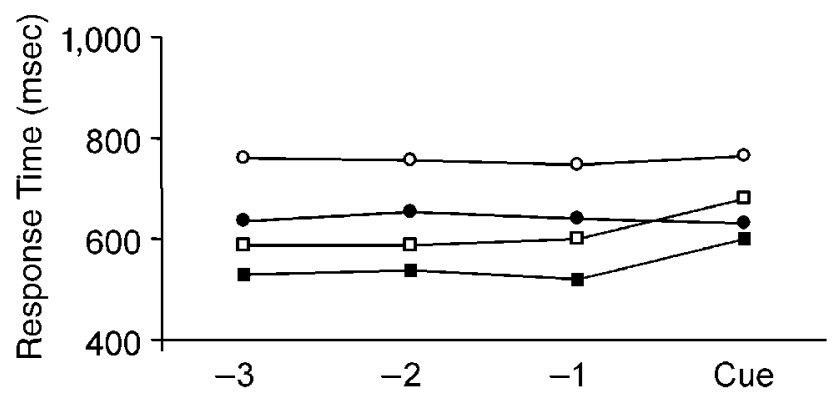

(B) Targets

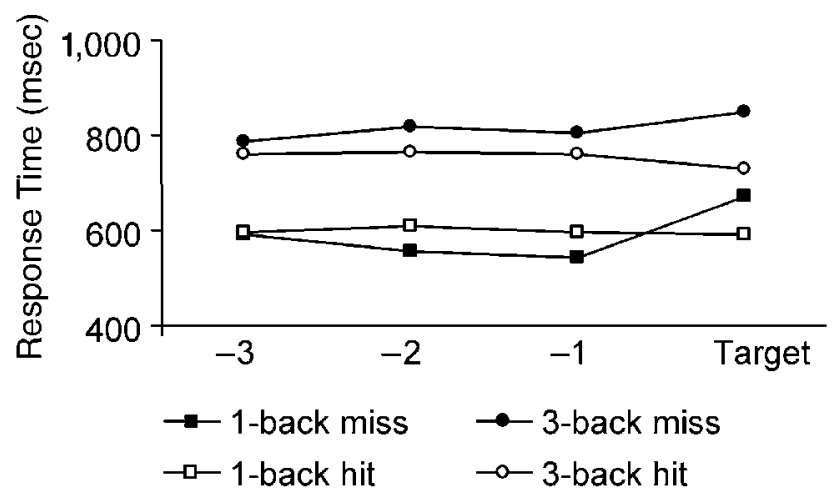

Figure 1. Mean response time for prospective cue (A) and $\boldsymbol{n}$-back target $(B)$ hits and misses and the three trials preceding hits and misses in Experiment 1.

novelty of this finding, Experiment 2 was designed to replicate this effect and to determine whether failures of PM arise from a complete disruption of preparatory attentional processes or from a more graded disruption of preparatory attentional processes. To examine this question, we had participants perform the $n$-back task with and without a PM load and compared RTs for trials preceding prospective misses with those for trials preceding stimuli that were matched to prospective cues in a no PM condition. If failures of PM result from a complete disruption of preparatory attentional processes, we expected RTs for trials preceding prospective misses to be similar to those for trials in the no PM load condition, where preparatory attentional processes were not required for task performance, which were matched to missed prospective cue trials; in contrast, if failures of PM result from only a partial disruption of preparatory attentional processes, we expected RTs preceding prospective misses to be slower than those for matched trials in the no PM load condition.

Experiment 2 was also designed to examine the failure to find an effect of $n$-back load on the success of PM, which was unexpected, given the findings that preparatory attentional processes demand WM capacity (Smith, 2003) and that occupying the central executive of WM disrupts PM (Marsh \& Hicks, 1998). One possibility for this null finding is that the disruptive effects of $n$-back load were offset by processing congruity between the on- going activity and PM cues (i.e., both involved processing letters), which is known to modulate the efficiency of prospective memory (Meier \& Graf, 2000). Therefore, in Experiment 2 the participants made $n$-back judgments for letters, and prospective cues were defined by color. This manipulation was intended to eliminate the influence of processing congruity.

\section{Method}

Participants. Eighteen individuals ( 8 female; $18-22$ years of age) participated in the study. They received course credit for completion of the study.

Materials and Procedure. The stimuli were identical to those in Experiment 1. The letters were presented in one of four colors (i.e., red, blue, green, or yellow). There were three types of trials (prospective cues on $10 \%$, targets on $30 \%$, and nontargets on $60 \%$ ). Identical stimulus lists were used for the PM and no-PM conditions.

The task design was a 2 (prospective load) $\times 2$ (n-back) factorial with five blocks for each condition (one practice block with 20 trials and four test blocks with 100 trials each). The four conditions were counterbalanced across participants. In the no-PM condition, the participants made $n$-back judgments for the letters and were told that letter color was irrelevant; in the PM condition, they made $n$ back judgments for most letters and prospective responses for letters that were presented in a color specified at the beginning of each block. Each color was used as a prospective cue once in the 1-back condition and once in the 3-back condition. For the no-PM load condition, the participants were instructed to press the $\mathrm{N}$ key if the current letter matched the $n$-back letter and the M key if it did not; for the PM load blocks, they were instructed to make $n$-back judgments for all letters other than those that were presented in the 
prospective color. The participants were also instructed that when a letter was presented in the prospective color, they were to respond by pressing the $\mathrm{V}$ key. A display indicating the prospective color for a given block was presented for $2 \mathrm{sec}$ at the beginning of each block (e.g., PM color = red) followed by a blank screen for $2 \mathrm{sec}$, then the first letter.

\section{Results}

For all analyses, the significance level was set at $p<$ .05 . In this experiment, there were effects of $n$-back load and prospective load on the proportion of correct responses. Prospective hits were more frequent in the 1back condition $(M=75 \%)$ than in the 3-back condition $[M=64 \%, t(17)=2.15]$. In an analysis including data for target and nontarget trials, there was an interaction between prospective load and $n$-back load $[F(1,17)=$ $6.87, \eta^{2}=.29$ ], with prospective load having little effect on performance in the 1-back condition (no-PM, $M=$ $84 \%$; PM, $M=86 \%$ ) and performance being reduced in the 3-back condition when a prospective load was added (no-PM, $M=72 \%$; PM, $M=68 \%$ ). PM load also interacted with stimulus $\left[F(1,17)=16.70, \eta^{2}=.50\right]$, being greater for targets (no-PM, $M=68 \%$; PM, $M=62 \%$ ) than for nontargets (no-PM, $M=89 \%$; PM, $M=92 \%$ ).

We evaluated predictions related to preparatory attentional processes and intention superiority in a series of analyses similar to those performed in Experiment 1. RT for prospective misses was faster than that for prospective hits $\left[F(1,17)=7.11, \eta^{2}=.30\right.$; see Figure 2] and for prospective misses $(M=680 \mathrm{msec})$ than for correct nontarget trials preceding target hits $(M=706 \mathrm{msec})$. This difference was not significant in the raw response time data $\left[F(1,17)=3.32, p>.08, \eta^{2}=.16\right]$ but was significant following log-transformation $[F(1,17)=7.68$, $\left.\eta^{2}=.31\right]$. RT was faster for trials preceding prospective misses than for trials preceding prospective hits $[F(1,17)=$ 13.26, $\left.\eta^{2}=.44\right]$. In an analysis comparing RT for trials

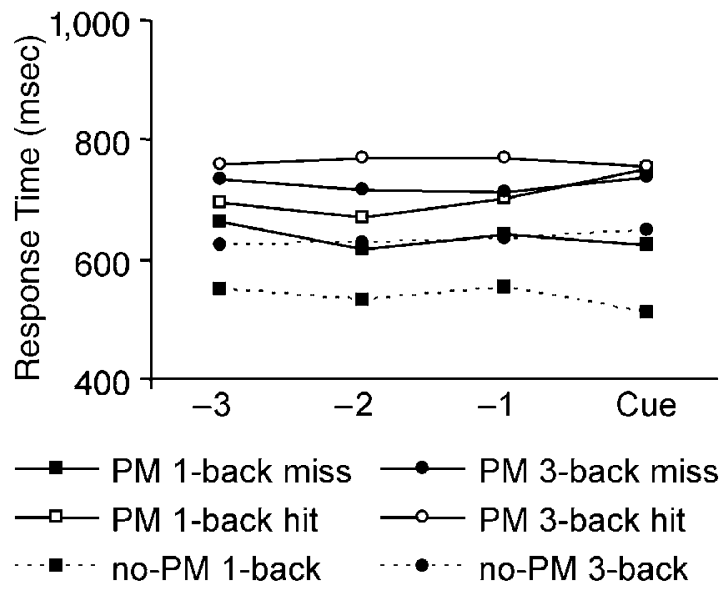

Figure 2. Mean response time for prospective cue hits and misses in the prospective load condition and matched trials in the no-prospective load condition, and the three trials preceding these trials in Experiment 2. preceding prospective misses with that for matched trials in the no-PM condition, the main effect of prospective load was significant $\left[F(1,17)=7.06, \eta^{2}=.29\right]$, with RT for trials preceding prospective misses being slower than that for trials in the no-PM condition. This finding indicates that failures of PM resulted from a graded rather than complete disruption of preparatory attentional processes.

We performed a series of analyses examining RT associated with target trials to examine the relationship between prospective load and $n$-back load. RT for target hits was modulated by prospective load $[F(1,17)=$ $\left.10.62, \eta^{2}=.39\right]$ and $n$-back load $\left[F(1,17)=5.73, \eta^{2}=\right.$ $.25]$, and these effects did not interact $(F<1)$. An analysis including trials preceding target hits and misses revealed an $n$-back load $\times$ accuracy $\times$ trial interaction $\left[F(2,34)=7.33, \eta^{2}=.30\right.$; see Figure 3$]$. In the 1-back condition, RT was faster for the two trials preceding misses than for the two trials preceding hits $[F(2,34)=$ $\left.4.55, p<.02, \eta^{2}=.21\right]$; in contrast, differences in RT preceding hits and misses in the 3 -back condition were not significant $\left[F(2,34)=2.85, p>.07, \eta^{2}=.14\right]$. These findings support those of Experiment 1 in demonstrating that different processes contributed to failures of $\mathrm{PM}$ and WM.

\section{GENERAL DISCUSSION}

This study was designed to examine whether faster RTs for prospective misses than for ongoing activity trials resulted from disruptions of preparatory attentional processes or from the expression of intention superiority. We predicted that disruptions of preparatory attentional processes would be associated with faster RTs for trials preceding prospective misses than for those preceding prospective hits (Smith, 2003). In contrast, intention superiority was expected to result in faster RTs that were limited to misses rather than extending over several trials (Marsh et al., 2002). The pattern of RT data across the two experiments provided support for both of these hypotheses. RT was faster for prospective misses than for ongoing activity trials, revealing an intention superiority effect (Marsh et al., 2002), and RT was faster for ongoing activity trials preceding prospective misses than for ongoing activity trials preceding prospective hits, which is consistent with the prediction that disruptions of preparatory attentional processes contribute to RT for prospective misses. In addition, RT for trials preceding prospective misses was slower than that for trials preceding matched stimuli in a no-PM load condition, indicating that failures of PM resulted from a graded rather than a complete disruption of preparatory attentional processes. This idea is consistent with other data indicating that the accuracy of PM varies over time (Maylor, 1996; West \& Craik, 1999) and the proposal that goal neglect contributes to disruptions of task performance in a variety of settings (Duncan, Emslie, Williams, Johnson, \& Freer, 1996). 


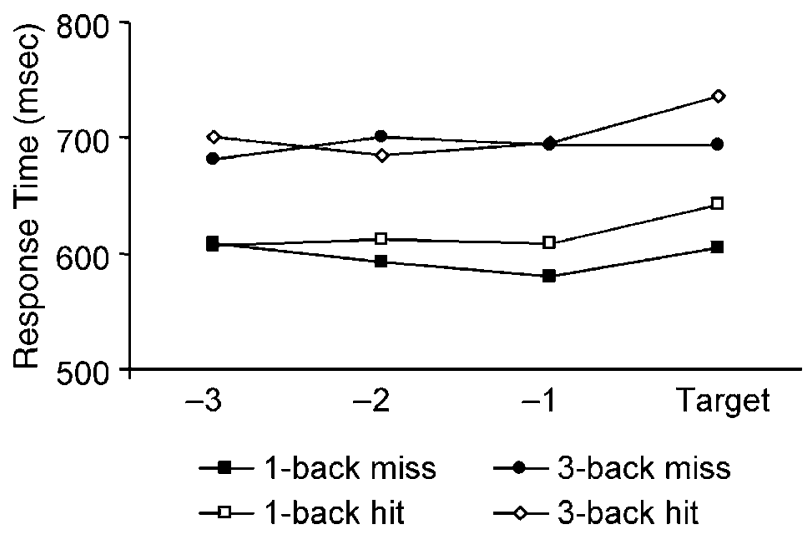

Figure 3. Mean response time for target hits and misses and the three trials preceding target hits and misses in Experiment 2.

$n$-Back load did not influence differences in RT for ongoing activity trials preceding prospective hits and misses, leading to the suggestion that disruptions of preparatory attentional processes were insensitive to the WM demands of the ongoing activity in these experiments. This finding was surprising, since preparatory attentional processes are thought to require the allocation of WM capacity (Smith \& Bayen, 2004) and individual differences in WM capacity correlate with RT measures of preparatory attentional processes (Smith, 2003). One possible explanation for this effect is that individual differences in WM capacity are important for the allocation of preparatory attentional processes, but they have relatively little effect on the maintenance of these processes over time. Consistent with this idea, Smith and Bayen (2004) found that instructing individuals to devote greater attention to the prospective or ongoing components of the task modulated preparatory attentional processes. Further research should provide insight into the dynamic relationship between WM capacity, the demands of the ongoing activity, and the allocation of preparatory attentional processes.

The frequency of prospective cues was higher in the present experiments than is typically the case in laboratory studies of event-based PM. This may lead one to wonder whether the present paradigm provides a valid index of PM or is more closely aligned with measures of WM or vigilance where target information is actively maintained in memory during task performance. We believe that a number of findings from this and other works indicate that individuals are not actively maintaining the prospective cues in memory in the current task. First, the RT profiles for trials preceding hits and misses for prospective cues and targets were dissimilar, with differences between prospective hits and misses being present at least three trials before cue onset and differences between target hits and misses varying with $n$-back load and not being present until one or two trials before target onset. If prospective misses resulted from failures to actively maintain the cues in memory, it seems that the RT profiles would have been more similar for prospective cue and target trials. Second, a recent study (West, Wymbs, Jakubek, \& Herndon, 2003) comparing the neural correlates of PM and target detection in the oddball taskwhich could be taken as a measure of vigilance - using a similar event rate (i.e., $5 \%$ cues) revealed distinct modulations of the event-related brain potentials differentiating oddball targets and prospective cues. Together, these findings indicate that individuals were not actively maintaining the prospective cues in memory, leading us to believe that the present paradigm provides insight into the nature of processes supporting PM that are distinct from those supporting WM or vigilance.

In summary, data from two experiments provide support for the idea that intention superiority and variation in the efficiency of preparatory attentional processes contributes to RT for missed PM cues (Marsh et al., 2002; Smith, 2003). Furthermore, variations in the likelihood, but not magnitude, of disruptions of preparatory attentional processes appear to be sensitive to the WM demands of the ongoing activity, at least when processing congruity between the ongoing activity and prospective components of the task is controlled. An important issue that remains to be resolved is the degree to which similar processes generalize to other settings where different types of PM cues and ongoing activities are used.

\section{REFERENCES}

BADDEley, A. (1986). Working memory. Oxford: Oxford University Press.

Duncan, J., Emslie, H., Williams, P., Johnson, R., \& Freer, C. (1996). Intelligence and the frontal lobe: The organization of goaldirected behavior. Cognitive Psychology, 30, 257-303.

ELLIS, J. (1996). Prospective memory or the realization of delayed intentions: A conceptual framework for research. In M. Brandimonte, G. O. Einstein, \& M. A. McDaniel (Eds.), Prospective memory: Theory and applications (pp. 1-22). Mahwah, NJ: Erlbaum.

Goschke, T., \& KunL, J. (1993). Representation of intentions: Persisting activation in memory. Journal of Experimental Psychology: Learning, Memory, \& Cognition, 19, 1211-1226.

MARSH, R. L., \& HicKs, J. L. (1998). Event-based prospective memory and executive control of working memory. Journal of Experimental Psychology: Learning, Memory, \& Cognition, 24, 336-349.

Marsh, R. L., Hicks, J. L., \& BinK, M. L. (1998). Activation of completed, uncompleted, and partially completed intentions. Journal of Experimental Psychology: Learning, Memory, \& Cognition, 24, 350-361.

Marsh, R. L., Hicks, J. L., Cook, G. I., Hansen, J. S., \& Pallos, A. L. (2003). Interference to ongoing activities covaries with the characteristics of an event-based intention. Journal of Experimental Psychology: Learning, Memory, \& Cognition, 29, 861-870.

Marsh, R. L., Hicks, J. L., \& Watson, V. (2002). The dynamics of intention retrieval and coordination of action in event-based prospective memory. Journal of Experimental Psychology: Learning, Memory, \& Cognition, 28, 652-659.

MaYlor, E. A. (1996). Age-related impairment in an event-based prospective memory task. Psychology \& Aging, 11, 74-78.

Meier, B., \& Graf, P. (2000). Transfer appropriate processing for prospective memory tests. Applied Cognitive Psychology, 14, S11$\mathrm{S} 27$.

Smith, R. E. (2003). The cost of remembering to remember in eventbased prospective memory: Investigating the capacity demands of delayed intention performance. Journal of Experimental Psychology: Learning, Memory, \& Cognition, 29, 347-361.

SMith, R. E., \& BAYEN, U. J. (2004). A multinomial model of event- 
based prospective memory. Journal of Experimental Psychology: Learning, Memory, \& Cognition, 30, 756-777.

Tulving, E. (1983). Elements of episodic memory. Oxford: Oxford University Press.

West, R., \& Craik, F. I. M. (1999). Age-related decline in prospective memory: The roles of cue accessibility and cue sensitivity. Psychology \& Aging, 14, 264-272.

WeSt, R., Wymbs, N., JAKUBeK, K., \& Herndon, R. W. (2003). Effects of intention load and background context on prospective remembering: An event-related brain potential study. Psychophysiology, 40, $260-276$.

\section{NOTE}

1. The trial preceding a target in the 1- and 3-back conditions was always a nontarget trial; therefore, response time (RT) for these trials was used as a comparison to RT for missed prospective cues that were also nontargets. These trials seem to serve as an appropriate baseline, given that prospective cue and target misses appear to be associated with variation in the efficiency of attentional processes that modulate task performance. Trials preceding prospective cues could be either targets or nontargets, making trials preceding prospective hits a less than optimal control.

(Manuscript received October 10, 2003;

revision accepted for publication August 17, 2004.) 